\title{
Scalar Dielectric Constant Extraction of Planar Materials from Reflection Measurements
}

\author{
Bektaş Çolak \\ Electrical \& Electronics Engineering, Alanya Alaaddin Keykubat Univertsity , Antalya, TURKEY
}

\begin{abstract}
Industrial plastics are widely used in warfare electromechanical systems to protect the internal electronic circuitry from environmental effects. Depending the purpose of the warfare system, the surface interaction with electromagnetic fields play a key role and the main parameter that determines reflection and the shielding of the electromagnetic fields from material surface is the dielectric constant of the material itself. In this paper, extraction of scalar dielectric constant from electromagnetic reflection measurement using an alternative two standard calibration technique in a low cost aluminum compact chamber is obtained. Calibration technique uses time domain measurements and converts them to frequency domain. Especially in R\&D phase of electromechanical integration of warfare systems, this technique is quite useful where the exact values of dielectric constants of planar industrial plastics are not required. Obtained reflection coefficient error level is $0.67 \mathrm{~dB}$ for $10 \mathrm{~mm}$ while it reaches $1.62 \mathrm{~dB}$ for $40 \mathrm{~mm}$ thickness materials. It is shown that calculated values are in good agreement with the reference measurement method performed by precision airline setup Agilent 85050C.
\end{abstract}

Keywords: Scalar Dielectric Constant, Planar Materials, VNA Reflection Measurement, Time Gating, Calibration.

\section{INTRODUCTION}

Characterization of dielectric materials can be performed by $\mathrm{S}$ parameters using Vector Network Analyzers (VNA). Measurements can be performed either in free space [1-5] or in coaxial or waveguide systems [6]. Each system has different sources of error [1]. Gating in the time domain is the most used technique to remove them. But it requires a careful investigation on the phase shift information of the reference plane. Also, the sample plate thickness is so important to define these phase shifts. In order to find alternative more accurate solutions, the new calibration techniques are developed for both measurement systems and VNA [7]. On the other hand, scalar network analyzer or tracking generator and spectrum analyzer combination can also be used [8]. All these techniques use $S_{11}$ and $S_{22}$. This paper presents an application of technique which uses only $S_{11}$ and two calibration standards for error reduction on the extraction of scalar dielectric constant in a low-cost compact screened test chamber [9-10]. The advantage of this technique is in simplicity originating from two calibration standards and free-space medium compare to near field medium [11]. The metallic screened test chamber is inexpensive due to its compact size and fixed position reference plane. Also because of the technique, the requirement of sensitivity to the reference plane phase shift information is removed. Measurements are done with a wide band horn antenna works in 2-18 GHz region. Since the details of the technique are given at [10]. It is focused in this paper to use this technique to extract the dielectric coefficient of planar samples. A brief information about the measurement system is given in section II. The basic set of extraction formulas are given in section III. Obtained results are given in section IV and a conclusion is followed.

\section{MEASUREMENT SETUP}

The material characterization chamber is given in Fig. 1. It has $2 \mathrm{~m} \times 1 \mathrm{~m} \times 1 \mathrm{~m}$ dimensions with a $2 \mathrm{~mm}$ aluminum shield to remove the interference from the outside environment in order to obtain a more silent test environment. The reflections are removed by polyurethane absorbers in the walls of the screen.

The distance between the reference plane and the antenna is given Fig. 2. Thanks to absorbers to decrease the aluminum wall reflections for easier interpretation of the results. 


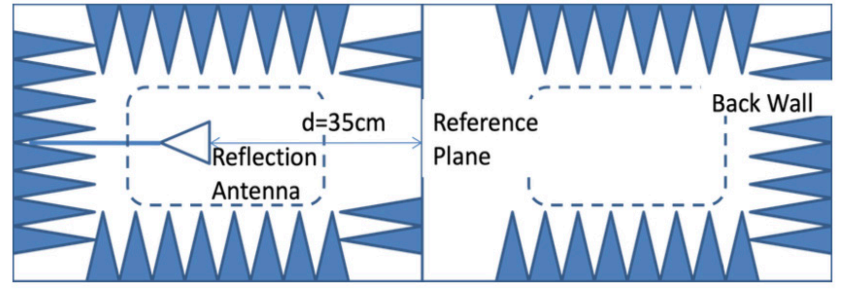

Figure1. Metallic compact material characterization chamber.

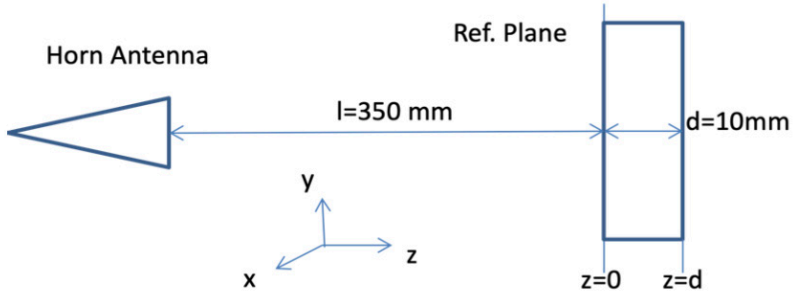

Figure 2. Distance and the geometry between the reference point and antenna.

\section{APPLIED TECHNIQUE}

The reference plane is the front face of the first calibration standard. Reflection occurs at both the front face and the back face of the planar sample. The total value of both reflections, $\Gamma_{d B}$, at the front face is given below [8].

$$
\Gamma_{d B}=20 \log _{10}\left(\frac{r_{12}\left(1-e^{-j 2 \Theta}\right)}{1-r_{12}^{2} e^{-j 2 \Theta}}\right)
$$

where,

$$
\begin{aligned}
& \Theta=k_{s} d \\
& k_{s}=\frac{2 \pi}{\lambda_{0}} \sqrt{\varepsilon_{r}} \\
& r_{12}=\frac{1-\sqrt{\varepsilon_{r}}}{1+\sqrt{\varepsilon_{r}}}
\end{aligned}
$$

The thickness is defined with $d$, the wavelength is given with $\lambda_{0}$ and dielectric constant is defined with $e_{r}$.

Since the samples are planar material it is well known that both edge reflections are canceled and add each other at the frequency spectrum periodically. Finally, it is obtained maximum and minimum points in the frequency spectrum. The extraction method is based on this fact.

We used the peak values while dip values also able to give this extraction. Peak values, $f_{\max }$, depend on the thickness and the dielectric constant of the planar sample.

$$
\left.f_{\max }\right|_{n}=\frac{c}{4 d \sqrt{\mathrm{e}_{r}}}(2 n+1) \quad n=0,1,2 \ldots
$$

where $\mathrm{c}$ is the speed of light.

Period of the peak values, $T$, easily calculated by subtracting two sequential peak or null points.

$$
T=\left(\left.f_{\max }\right|_{n=0}\right)-\left(\left.f_{\max }\right|_{n=1}\right)
$$

If we organize (4) by using (3), dielectric constant will be obtained.

$$
\varepsilon_{r}=\left(\frac{c}{2 d T}\right)^{2}
$$

After performing the reflection measurement, the dielectric constant is firstly calculated by (5). This first calculation includes measurement errors that originated from the mentioned calibration process [10]. Error level of extracted dielectric constant highly dependent to the error level of the measured reflection coefficient results since the period of the maximum points is obtained through these measurements. Therefore, the calibration process plays an important role in the whole system. Since the calibration technique [10] is based on the two standards, it is necessary to compare the calculated results with measured ones. The difference between them is assigned as error term $e_{k^{\prime}}$ (in $\mathrm{dB}$ ).

$$
e_{k}=\left|R_{d B}-\Gamma_{d B}\right|
$$

For the whole range of the measurement, an average term is given as below by using each error term at frequency points $(k=1 . . i)$

$$
e_{T}=\sum_{k=1}^{i} \frac{e_{k}}{i}
$$

\section{RESULTS}

Four different thickness polypropylene plates are used in measurements and each one error analysis is done. As a comparison, a small part from the dielectric plate is machined in a toroid shape to insert in a precision airline setup namely Agilent 85050C coaxial material characterization kit and the dielectric constant is measured averagely between $5 \mathrm{GHz}$ to $15 \mathrm{GHz}$ as 2.20 . For each thickness dielectric constant is calculated from the measured values with (5) by minimizing the error term. Theoretical values of dielectric constant are obtained by using (1). Found dielectric constant values are given for each thickness in Fig. 3-6.

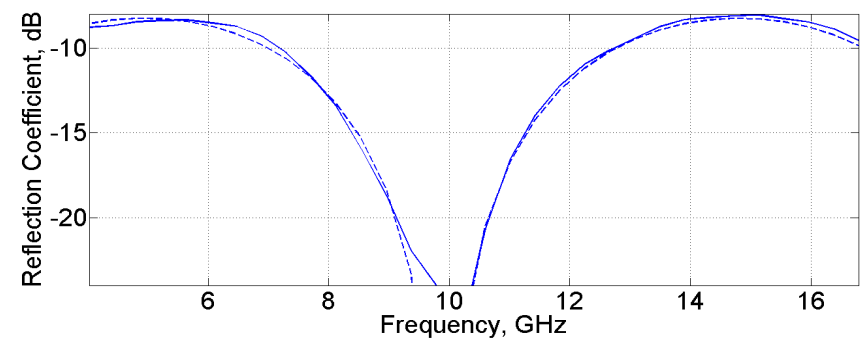

Figure 3. Calculated reflection coefficient for the parameters of $d=10 \mathrm{~mm}$ (Solid: measured, dashed: theoretical). The total error is minimum for $\varepsilon_{\mathrm{r}}=2.26$ and equal to $0.67 \mathrm{~dB}$

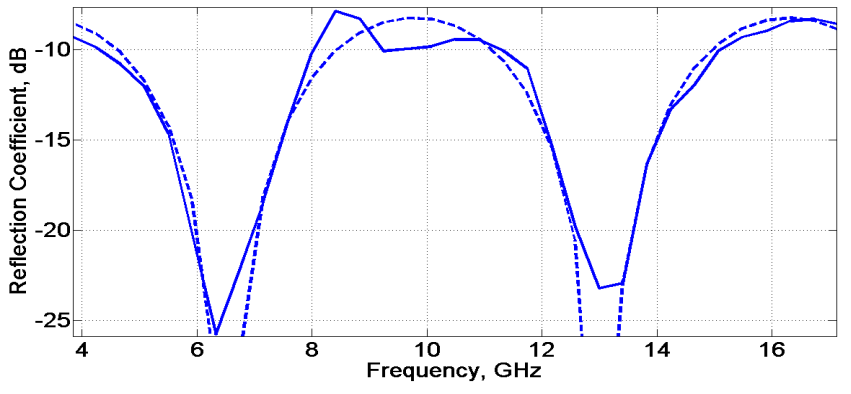

Figure 4. Calculated reflection coefficient for the parameters of $d=15 \mathrm{~mm}$ (Solid: measured, dashed: theoretical). The total error is minimum for $\varepsilon_{\mathrm{r}}=2.25$ and equal to $1.37 \mathrm{~dB}$ 


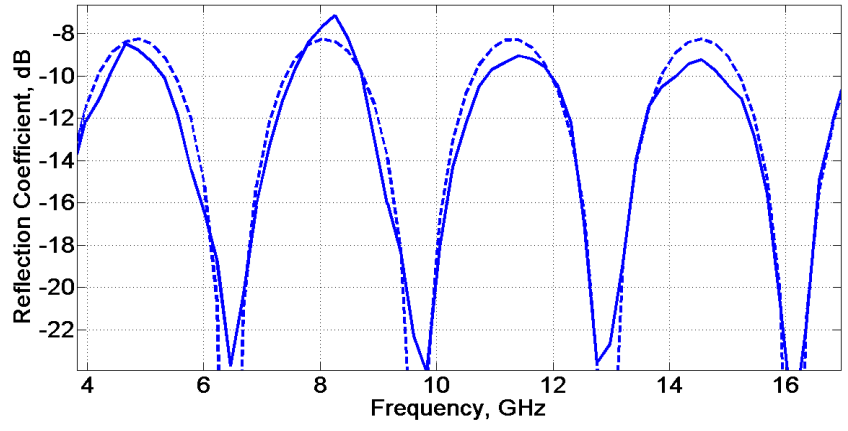

Figure 5. Calculated reflection coefficient for the parameters of $d=30 \mathrm{~mm}$ (Solid: measured, dashed: theoretical). The total error is minimum for $\varepsilon_{\mathrm{r}}=2.25$ equal to $1.51 \mathrm{~dB}$

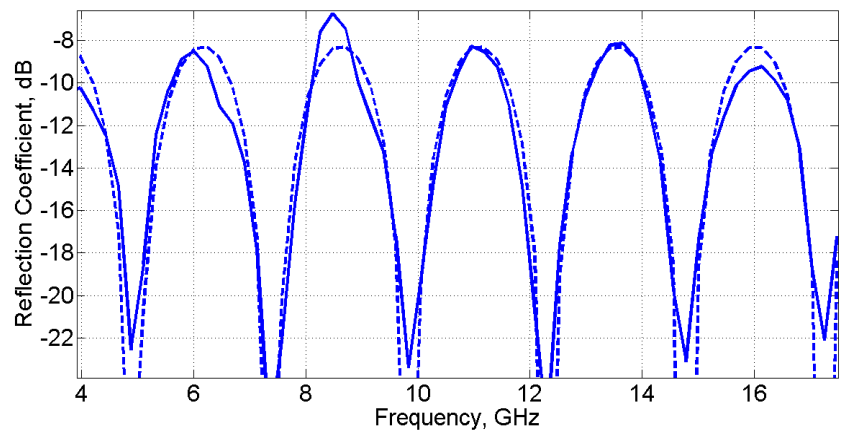

Figure 6. Calculated reflection coefficient for the parameters of $d=40 \mathrm{~mm}$ (Solid: measured, dashed: theoretical). The total error is minimum for $\varepsilon_{\mathrm{r}}=2.24$ equal to $1.62 \mathrm{~dB}$

It is shown that calculated values are in good agreement with the reference measurement method performed by precision airline setup Agilent 85050C. Error is rising by increasing the thickness of the sample because the peak and null numbers in the specified wideband are increasing. Since the dielectric constant is extracted from this information, the measurement is more sensitive to the at higher thickness values.

\section{CONCLUSION}

A technique is proposed to be used on the extraction of scalar dielectric constant from reflection measurement using an alternative two standard calibration in a low-cost compact chamber. Advantage of this technique is in simplicity and reducing the number of calibration standards. It is simple because, there is no need to consider the possible gating errors originating from the positioning of the sample. Especially in R\&D phase of electromechanical integration of warfare systems, this technique is quite useful where the exact values of dielectric constants of planar industrial plastics are not required. This technique is quite useful where the exact values are not required. Obtained reflection coefficient error level is $0.67 \mathrm{~dB}$ for $10 \mathrm{~mm}$ while it reaches $1.62 \mathrm{~dB}$ for $40 \mathrm{~mm}$ thickness materials. Extracted dielectric constant is in good agreement with the theoretical value. Validation of the technique is done by a precision airline setup namely Agilent $85050 \mathrm{C}$. It is shown that the calibration technique can be used for the extraction of the dielectric constant of planar materials especially in fast $R \& D$ phase of the electromechanical systems.

\section{REFERENCES}

[1] Smith, F.C., Chambers, B., \& Bennett, J.C. (1994). Methodology for Accurate Free-space Characterisation of Radar Absorbing Materials, Proc. IEE Science measurement and Technology, 141(6): 538-546

[2] Gu, D., Houtz, D., Randa, J., \& Walker, D. K. (2011, July). Extraction of reflectivity from microwave blackbody target with free-space measurements. In 2011 IEEE International Geoscience and Remote Sensing Symposium (pp. 3847-3850). IEEE.

[3] Bartley, P. G., \& Begley, S. B. (2012, May). A new free-space calibration technique for materials measurement. In 2012 IEEE International Instrumentation and Measurement Technology Conference Proceedings (pp. 47-51). IEEE.

[4] Kemptner, E., \& Thurner, S. (2012, March). Free space material characterization for microwave frequencies. In 20126 th European Conference on Antennas and Propagation (EUCAP) (pp. 3513-3515). IEEE.

[5] Rocha, L. S., Junqueira, C. C., Gambin, E., Vicente, A. N., Culhaoglu, A. E., \& Kemptner, E. (2013, August). A free space measurement approach for dielectric material characterization. In 2013 SBMO/IEEE MTT-S International Microwave \& Optoelectronics Conference (IMOC) (pp. 1-5). IEEE.

[6] Caijun, Z., Quanxing, J., \& Shenhui, J. (2011). Calibration-independent and position-insensitive transmission/reflection method for permittivity measurement with one sample in coaxial line. IEEE Transactions on Electromagnetic Compatibility, 53(3): 684-689.

[7] Hasar C. U. (2008). A New Calibration-Independent Method for Complex Permittivity Extraction of Solid Dielectric Materials, IEEE Microwave and Wireless Component Letters, 18(12): 788-790

[8] Hasar, U. C. (2008). A fast and accurate amplitude-only transmission-reflection method for complex permittivity determination of lossy materials. IEEE Transactions on Microwave Theory and Techniques, 56(9): 2129-2135.

[9] Colak, B. (2016, June). A comparative study on the calibration of Frequency domain reflection measurements for a compact test chamber. In 2016 9th International Kharkiv Symposium on Physics and Engineering of Microwaves, Millimeter and Submillimeter Waves (MSMW) (pp. 1-4). IEEE.

[10] Colak B., Helhel S. (2019). A new error reduction technique for reflection coefficient measurements for use in quick laboratory tests, International Journal of Electronics, 106(2): 237-249

[11] Wu, S. M., Guan, S. W., Li, C. D., Tsai, L. X., Kuo, C. T., Hsieh, M. K., \& Su, C. H. (2018, May). Dielectric constant and loss-tangent extraction using near-field technology and phase delay method. In 2018 IEEE International Symposium on Electromagnetic Compatibility and 2018 IEEE Asia-Pacific Symposium on Electromagnetic Compatibility (EMC/APEMC) (pp. 683-686). IEEE. 